\title{
The rationale and design of the minocycline plus amiodarone versus amiodarone alone (MINAA) trial for the prevention of atrial fibrillation after cardiac surgery
}

\author{
Abdallah Khaled Alameddine ${ }^{1 *}$, Paul Visintainer ${ }^{2,3}$, Victor Alimov ${ }^{1}$, Jennifer Germain ${ }^{1}$, \\ Yvonne A. Alameddine ${ }^{1}$, Daniel Engelman ${ }^{1}$, John A. Rousou ${ }^{1}$ \\ ${ }^{1}$ Divisions of Cardiac Surgery, Baystate Medical Center, Springfield, USA \\ ${ }^{2}$ The Department of Epidemiology and Academic Affairs, Springfield, USA \\ ${ }^{3}$ Tufts School of Medicine, Boston, USA \\ Email: ${ }^{*}$ akalameddine@gmail.com
}

Received 4 August 2012; revised 6 September; accepted 16 September 2012

\begin{abstract}
Objectives: Amiodarone administration is presently considered in the prevention of new-onset postoperative atrial fibrillation (PAF) after cardiac operations, but relapse of PAF requiring anticoagulation therapy at hospital discharge is prevalent despite amiodarone prophylaxis. PAF is also associated with increased morbidity and mortality including complications resulting from long-term anticoagulation therapy. Currently, the most effective therapy to prevent PAF after cardiac surgery remains undetermined. Inflammatory mechanisms may be partly responsible for PAF. Minocycline, a tetracycline antibiotic, has specifically an atrial myocytes anti-apoptotic effect, decreases right atrial tissue inflammation and oxidative stress activity. These observations led to this trial's hypothesis that the addition of minocycline to amiodarone may favorably affect suppression of PAF. Methods: This trial compares the efficacy and safety of minocycline plus amiodarone versus amiodarone alone, in the prevention of PAF among adult patients undergoing cardiac procedures. All patients receive Beta-blocker agent. The primary outcome is PAF occurrence. Secondary outcomes include thromboembolic stroke, need for pharmacologic or electric cardioversion, mediastinal exploration for sepsis or for anticoagulation-related bleeding, serious drug side effects, length of hospital stay and 30-day mortality from cardiovascular causes. Results: This is an ongoing prospective single center randomized controlled clinical trial. Conclusion: The trial provides information on the comparative effectiveness of this low-risk prevention therapy of PAF that could be integrated in clinical practice. (Clinicaltrials.gov number, NCT 01422148).
\end{abstract}

${ }^{*}$ Corresponding author.
Keywords: Arrhythmias; Clinical Electrophysiology; Drugs; Cardiovascular Pharmacology; CV Surgery: Coronary Artery Disease; CV Surgery: Valvular Disease; Thrombosis Risk Factors

\section{TRIAL OBJECTIVES}

At present, the average PAF rate, using amiodarone monotherapy prophylaxis, is about $26 \%$ based on this institution's current incidence of PAF and those of pooled published studies that tested efficacy of conventional amiodarone prophylaxis in adult cardiac operations performed "on" or "off-pump".

The primary aim of the trial is to evaluate the efficacy of minocycline - a drug that inhibits inflammation - combined with amiodarone, as prophylaxis in reducing PAF following cardiac operations performed on persons 18 years of age or older. The secondary aim is to establish minocycline safety. Examples of cardiac surgery include coronary artery bypass grafting (CABG), heart valve replacement/repair, or combination of $\mathrm{CABG}$ and valve surgery.

\section{RATIONALE}

Depending on the cardiac procedure performed, and without drug prophylaxis, PAF affect approximately $15 \%$ to $65 \%$ of cardiac surgery patients $[1,2]$. The majority of the initial episodes of PAF generally occurs during the first two to five days after cardiac procedures, but PAF can occur at any point in the first two weeks after the recovery period. PAF can be associated with high morbidity and long-term mortality; including development of thromboembolic strokes, increased need for inotropic support, reoperation for bleeding, prolonged hospital stay, and increased readmission rate and increased overall health care costs. 
The likelihood that PAF will develop following cardiac operations depends on a variety of initiating risk factors regardless whether the procedure is done "on" or "off-pump" [3]. These independent predictors are older age $>70$ years, NYHA functional class heart failure, left ventricular ejection fraction $\leq 35 \%$, prolonged aortic cross-clamping time, unstable angina, ischemic myocardial injury, serious co-existing conditions, previous history of PAF, atrial manipulation, and red cell transfusion $[4,5]$.

The contemporary therapeutic options for the prevention of PAF proposed in most studies include amiodarone with or without use of $\beta$-blockade therapy. However, despite amiodarone prophylaxis, PAF still occurs in up to $35 \%$ of cases [6-14]. The range of the response rate to amiodarone prophylaxis is wide because of variability of dose, timing of initiation and duration of treatment, and drug side-effects.

In the trial by Zebis [11] and colleagues, among 113 patients who received higher dose of amiodarone 8550 $\mathrm{mg}$ total postoperative dose given over 5 day-adverse events led to discontinuation of this drug in $3(2.7 \%)$ patients and $2(1.8 \%)$ patients required short-term pacemaker insertion because of severe bradycardia. Similarly, although the trial by Yagdi and colleagues [12] has reported the lowest atrial fibrillation incidence (10.4\%) with amiodarone prophylaxis after $\mathrm{CABG}$, in that study however amiodarone had to be discontinued in $4(5.2 \%)$ patients because of slow heart rate $<60$ beats/min or prolonged QT interval $>440 \mathrm{~ms}$ and $3(3.9 \%)$ patients required pacing. With somewhat lower prevention rate of post operative atrial fibrillation after CABG/valve repair or replacement/ combination procedures, the PAPABEARtrial [13] "Prophylactic oral amiodarone for the prevention of arrhythmias that begin early after revascularization, valve replacement, or repair" has reported bradycardia occurring in $34 / 299(11.4 \%)$ of cases for presumed side effects and requiring amiodarone dose reduction, following a total amiodarone administration of 10 $\mathrm{mg} / \mathrm{kg} /$ day given for 13 days. Given these results, it seems wise to lower the total dose of amiodarone or to avoid its use altogether in older patients ( $\geq 70$ years) especially if severe renal or pulmonary impairments are present.

The treatment period and drug dosing for amiodarone prophylaxis have varied among pooled data published in twenty eight trials assessing various drug regimens [4]. The efficacy of amiodarone as a prophylactic drug, given either intraoperatively or preoperatively, in the reduction of PAF is somewhat limited. Furthermore, the recommendation of a time delay in given the drug is problematic because it is impractical to implement for the more urgent or emergent cardiac surgery cases.

One of the lowest frequency (10.4\%) of PAF after cardiac surgery was reported by Yagdi and associates
[12]. In that study, which included selected cohorts of patients for coronary revascularization alone, thus excluding heart valve operations, 77 patients received 10 $\mathrm{mg} / \mathrm{kg} / \mathrm{d}$ of intravenous amiodarone postoperatively for 48 hours, then a total dose of $9 \mathrm{~g}$ of oral amiodarone given over 30 days after the operation. In the PAPABEAR-trial, which is considered the largest controlled trial, the incidence of PAF was reduced to $16.1 \%$ using a regimen of amiodarone $(10 \mathrm{mg} / \mathrm{kg} /$ day $)$ administered 7 days prior to surgery and then continued for 6 more days after the operation. Criticism of that study include unrealistic preoperative dosing because it is not applicable or achievable in urgent or emergent condition settings. The rate of prophylactic failure was highest in the $\mathrm{ARCH}$ study [14]. In that study atrial fibrillation occurred in $67 / 142(47 \%)$ of platients on placebo versus $56 / 158$ $(35 \%)$ on amiodarone $(p=0.01)$, representing a $26 \%$ relative reduction. Their results with low prevention rate are explained by the shorter period of administration of amiodarone given ( $1 \mathrm{~g} /$ day/ for 2 days), the higher number of patients with prior myocardial infarction (35\%), the many patients were identified as urgent candidates for bypass, and the larger number of older patients aged $\geq 70$ years $(33 \%)$.

All of these reports suffer from several common potential limitations. Therefore, at present, the most efficacious perioperative drug therapy protocol to reduce PAF remains undetermined and, accordingly, there is a need for a new drug therapy that confers protection against PAF.

\section{CAUSES OF PAF}

Although the pathogenesis of PAF after "on" or "offpump" has not been fully elucidated and may have multiple causes, recent work show that inflammation and cellular apoptosis of atrial myocytes play an important role. Increased magnitude of inflammatory biomarkers such as C-reactive protein, high molecular indices of apoptosis in right atrial tissues, exagerated oxidative stress and atrial myocytes necrosis, all predict the development of PAF [15-17]. The findings that the complement system and C-reactive protein, both acute phase reactants produced in response to inflammatory cytokines are activated in the systemic inflammatory response to $\mathrm{CPB}$, strengthen the argument for the role of inflammation in PAF. In addition, the effects of reactive oxygen species scavengers and antioxidant administration have all been examined in patients undergoing $\mathrm{CABG}$ operations and found to be significant. Finally, recent data from randomized controlled trials on the prevention of PAF found that steroidal or non-steroidal drugs with anti-inflammatory activities such as corticosteroids and statins, or antioxidants (e.g., vitamine E), 
administered during cardiac surgery, all reduced the incidence of PAF [18-20].

Collectively, these observations argue that a potential approach to suppression of PAF could be achieved by altering atrial inflammation or components of the apoptotic machinary.

Minocycline is a semisynthetic second-generation tetracycline with a long half-life (11 - 18 hours), and acceptable side-effect profile. Minocycline is a well established bacteriostatic antibiotic which is primarily used in humans to treat acne and other skin infections. Minocycline exerts significant anti-inflammatory effects through blockade of nuclear factor- $\mathrm{kB}$ nuclear translocation, has inhibitory activity against apoptosis via attenuation of oxidative stress induced by tumor necrosis factor- $\alpha$, up-regulates mitochondrial bcl-2 expression, and reduces mitochondrial calcium uptake and calcium-induced cytochrome $\mathrm{C}$ release which leads to a downregulation of pro-inflammatory cytokine output [20-24]. Furthermore, minocycline-mediated neuroprotection has been extensively reported and found to protect the brain from hypoxia-ischemia injury [23]. Similarly, significant beneficial effects of minocycline as a cardioprotective agent has been shown during ischemia/reperfusion injury in primary cultures of myocytes and in the intact heart. A recent in-vitro study found that minocycline directly inhibits poly (ADP-ribose) polymerase-1 (PPAP-1) enzymatic activity in a cell free culture; PPARP-1 is known to mediate apoptosis.

\section{AMIODARONE DOSING}

The perioperative amiodarone drug regimen that was implemented in this trial is based on inferences from randomized and observational studies, all of which have reported encouraging results [10-14,25]. Furthermore, based on a recent meta-analysis of 19 randomized controlled trials conducted by Bagshaw and associates [7], evaluating the effect of amiodarone for prevention of PAF after cardiac surgery, suggested that a $5-10 \mathrm{~g}$ dose over 2 week-period is sufficient.

\section{TRIAL DESIGN AND OVERSIGHT}

This is a single-blinded randomized study conducted on patients18 years of age or older, who are undergoing cardiac surgery with no symptoms of atrial fibrillation in the 6 months before randomization. All eligible patients are randomly assigned to receive therapy with either intravenous minocycline (Minocin, Triax Pharmaceuticals) at a dose that was suggested by that company, of $100 \mathrm{mg}$ daily $\times 5$ starting intra-operatively in addition to standard of care amiodarone, or amiodarone monotherapy and followed up with 2 visits, at 2 weeks and 6 weeks after hospital discharge for routine post-operative management. Eligible patients are randomized in the operating room. All patients receive intraoperative amiodarone 150 $\mathrm{mg}$ slow intravenous bolus dose. This dose is repeated, if needed, for tachyarrhythmias within the first 24 hours after the operation. All patients are then switched to oral amiodarone $400 \mathrm{mg}$ twice daily on post operative day 1 for 7 days and then $200 \mathrm{mg}$ twice daily for the next 7 days. Beta-blocker (Metoprolol) is also given according to our Hospital protocol. The primary end point is the occurrence of PAF, which is defined as a paroxysmal disorder characterized by rapid, irregular electrical activity of the atria requiring chemical or electrical cardioversion, irrespective of extent, during the postoperative period including the 6-week surveillance period after hospital discharge.

\section{POST OPERATIVE ASSESSMENT FOR PAF}

Telemetry monitoring is used for hospital in-patients, with follow-up office 12-lead electrocardiogrphic rhythm recordings at 2 weeks after hospital discharge. The reason for not using the Holter monitor is mainly to limit the cost of the study.

\section{EFFICACY EVALUATION}

The primary outcome includes the interim occurrence of PAF. Secondary outcomes includes a 30-day hospital mortality from cardiovascular causes, hospitalization for cardiovascular events, need for chemical or electrical cardioversion, thromboembolic stroke, anticoagulation requirement for persistent PAF, resternotomy for mediastinal sepsis, and for bleeding related to anticoagulation or cardiac tamponade. Primary efficacy will be analyzed by intention to treat depending on the assignment at randomization. An independent safety and monitoring board (an appointed staff cardiologist, unaware of the treatment assignments) will adjucate all primary and secondary outcomes and will monitor trial operation.

\section{SAFETY AND TOLERABILITY}

Are assessed on the basis of the incidence of new symptoms or treatment discontinuation of study drug because of side effects. An adverse effect is classified as serious if patient requires hospitalization. Hypersensitivity reactions directly related to minocycline can include anaphylaxis and hypotension; wheezing, bronchial spasm, shortness of breath, eosinophilic pneumonia; sensation of burning, difficulty swallowing, exanthema, angioedema, nausea, vomitting, or diarrhea. Liver toxicity has also been reported.

Amiodarone may cause hypotension, bradycardia, pulmonary fibrosis, thyroid abnormalities, blue tinged skin coloring, or delirium. For the main analysis, participants who are randomly assigned to receive minocycline and 
amiodarone are compared with participants who are randomly assigned to amiodarone.

Is minocycline safe in such cases? Minocycline usage is much the same as tetracycline antibiotics. Immediate hypersensitivity reactions that are directly attributable to minocycline allergy are rare. It is hypothetized that the rate of discontinuation of study drug, because of unanticipitated troublesome drug side effects, would be low and similar in the two groups. The combined and concomitant effects of Mincycline and Amiodarone on liver function are unknown; therefore AST \& ALT laboratory tests will be checked pre-operatively, post-operatively at 24 hours and 96 hours, and at discharge.

\section{STUDY CONDUCT}

Initial screening and eligibility are performed by the PI, a research nurse, or by the co investigators. The trial protocol has already been approved by this Hospital's Institutional Review Board (IRB ID \#134759). Written informed consent will be obtained from all patients.

\section{PATIENT POPULATION}

\subsection{Eligible Subjects}

Willing are also willing to participate for the duration of the trial (6 weeks). All non-congenital cardiac operations are included: Coronary artery bypass graft (CABG), valve repair/replacement, or combination of $\mathrm{CABG}$ and heart valve operations.

\subsection{Exclusion Criteria}

Includes prior (within 6 months) or current PAF or flutter, patients undergoing concommitant surgical $\mathrm{AF}$ ablation for a history of AF, prior cerebrovascular event, cardiogenic shock or resuscitation, evidence of hepatic or renal dysfunctions (i.e., an alanine aminotransferase level that is $\geq$ twice the upper limit of the normal range, or either a serum creatinine level that is $\geq 2.0 \mathrm{mg} / \mathrm{dL}$ or need for preoperative dialysis), thyrotoxicosis, pregnancy, severe COPD (FEV1/FVC $<70 \%$ ), recent history of drug or alcohol abuse, and intolerance to tetracycline or amiodarone. Finally, because a core scientific basis of the trial concerned the role of underlying atrial tissue inflammatory activity, patients with inflammatory conditions such as lupus, severe arthritis, thyroiditis or inflammatory bowel disease are excluded; as are patients taking preoperative immunosuppressant agents, long-term oral corticosteroids, or estrogen replacement; and patients with a newly diagnosed cancer ( $<5$ years) are also excluded.

\section{RANDOMIZATION}

Assignments are prepared centrally by the pharmacist.
Since patients are unaware of the results of treatment arm, personnel involved in the study are asked not to disclose the treatment type to eligible study patients. All patients will receive Amiodarone per protocol, not allowing any apparent difference in treatment between patients. Staff will be inserviced throughout the length of the protocol to maintain patient blinding of study treatment.

A sequential numbering code will be used to de-identify subjects. Clinical data are collected and entered into an electronic data base by the principal investigator, coinvestigator, and by the research nurse. After 6 week follow-up, outcomes for the group receiving amiodarone and minocycline are compared with outcomes for group receiving amiodarone and placebo. Confidentiality of medical records will be maintained in an encrypted password protected Baystate computer and de-identified by sequential numbering. Access to patients' files is limited to research staff. These files will be maintained for 2 years after the completion of the study. The de-identified trial data will be utilized for publication. The CONSORT flow diagram for reporting Randomized Controlled Trials will be followed (see flow diagram).

The covariates of interest and outcomes are collected prospectively on a standardized data form. Standard definitions for disease severity criteria and for patient variables and risk factors of outcomes are those defined and identified by the STSACD as a risk factor in the published STS mortality and morbidity risk models version 26.8 accessed July 18th, 2009

at www.sts.org/sections/stsnationaldatabase) that include information about patient demographics such as age, gender; baseline cardiac data and coexisting conditions such as hypertension, diabetes mellitus, body-mass index $\geq 30 \mathrm{~kg} / \mathrm{m}^{2}$, history of smoking, cerebrovascular disease, peripheral vascular disease, hyperlipidemia, COPD, recent myocardial infarction ( $<30$ days), angiographic evidence of coronary artery disease, angina pectoris, coronary intervention, presence of shock and preoperative status; echocardiographic indices such as ejection fraction, type of mitral/aortic valve disease, mean aortic/mitral valve area/gradient, pulmonary artery systolic pressure, left atrial antero- posterior diameter $\geq 4.5 \mathrm{~cm}$, NYHA class, and left ventricular end-systolic/end-diastolic dimmensions as determined by Doppler assessment of LV diastolic function.

Surgical procedures include use of CPB or "off-pump" approach, valvular repair/replacement, $\mathrm{CABG}$, or combined $\mathrm{CABG} /$ heart valve procedures; operative data such as duration of CPB or aortic cross-clamping, blood loss and amounts of packed red blood cell or platelet trans- fused, pharmacotherapy at time of randomization (broadly categorized into $\beta$-blockers, statins, calcium channel blockers, diuretics, angiotensin converting enzyme inhibitors, and aspirin.

Postoperative data include percentage of patients in 


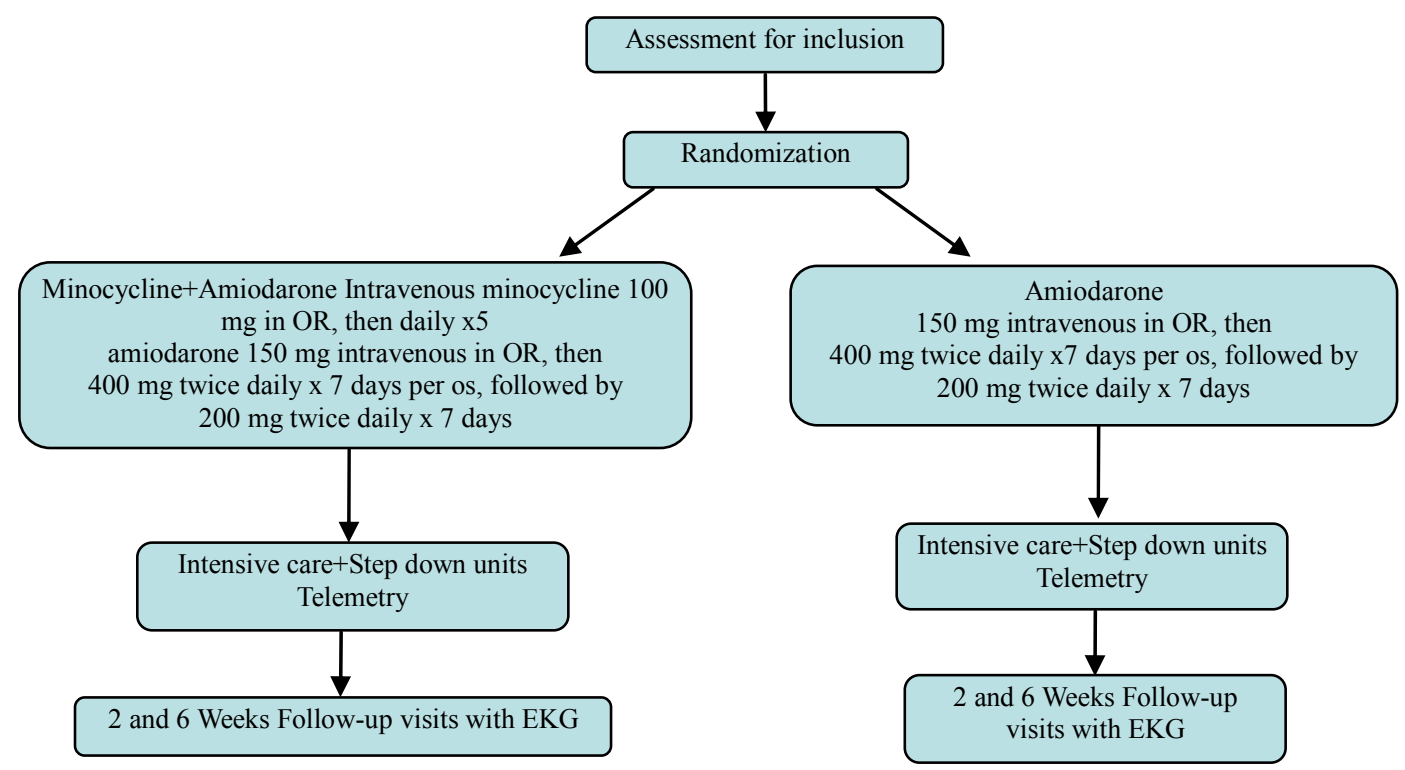

Flow diagram

PAF requiring therapy (anticoagulation, cardioversion, and need for additional anti-arrhythmic drugs to restore sinus rhythm), thromboembolic stroke, readmission for PAF; resternotomy for mediastinal bleeding or cardiac tamponade, paracentesis thoracis or tube thoracostomy (to evacuate hemorrhagic pleural effusions), minocycline or amiodarone drug related adverse effects necessitating discontinuation of treatment, mediastinal wound sepsis, pneumonia, hospital mortality from cardiac causes and organ failure.

Hospital mortality is defined as death from cardiac causes during the same hospitalization or within 30 days of procedure. Cardiac complications are defined as need for intraaortic balloon pump or inotropic support for $>24$ hours, increase of creatine kinase-myocardial band $(>3 \mathrm{x})$ serum level, or decrease of cardiac output $\leq 1.8 \mathrm{~L} / \mathrm{min} / \mathrm{m}^{2}$. Pulmonary complications are defined as prolonged $(>24$ hours) mechanical ventilation. Stroke is defined as neurologic central deficit lasting $>72$ hours. Renal dysfunction is defined as increase by $>50 \%$ of preoperative creatinine level or requirement for dialysis.

\section{SURGICAL TECHNIQUE}

Cardiac operations are done according to the standard practice utilizing either minimally invasive approach "offpump" or CPB “on-pump". Intravenous cefazolin antibiotic prophylaxis is administered (or vancomycin and gentamycin for penicillin allergic patients). Anesthesia is induced with fentanyl, etomidate, midazolam, and pancuronium and maintained with fentanyl, and isoflurane supplemented with sevoflurane. Conventional approach is followed including midline sternotomy, and when $\mathrm{CPB}$ is used it is initiated through cannulation of the right atrium- or if required bicaval venous cannulationand ascending aorta. The circuit includes membranous oxygenator, arterial filters, warm antegrade and retrograde blood hyperkalemic cardioplegia and moderate systemic hypothermia $\left(32^{\circ} \mathrm{C}\right)$.

A cell saver suction device is used to process blood remaining in the cardiotomy reservoir at the end of operation. Direct aortic clamping is utilized, and the pump flow is set between 2.2 and $2.4 \mathrm{~L} / \mathrm{min} / \mathrm{m}^{2}$. The perfusion pressure is maintained between 70 and $90 \mathrm{mmHg}$. Antifibrinolytic agent aminocaproic is administered depending on the preoperative creatinine level. Off-pump CABG is performed with cardiac positioning and coronary artery stabilizing devices. Serum glucose levels are maintained $<150 \mathrm{mg} / \mathrm{dL}$ with continuous insuline infusion.

Subsequent care in the cardiac surgical unit follow standard practice including analgesics, correction of electrolytes abnormalities, intravenous fluids for volume, blood or blood product transfusions, and sedatives and weaning of mechanical ventilation. Inotropic support and vasoactive agents are given as needed guided by physiologic hemodynamics, and postoperative $\beta$-blockeroral metoprolol $25 \mathrm{mg}$ twice daily - is given to all patients for rate control. Chest tubes are removed on the first postoperative day if the drainage is $<20 \mathrm{cc}$ per hour. Adequate blood oxygenation and urine output are maintained. Unless atrio-ventricular block exists, the temporary pacer wires are removed on the $3^{\text {rd }}$ postoperative day, and the patients are moved to a step-down unit with telemetry or Holter monitoring. A specific protocol careset intended for rapid response PAF (ventricular response $>120$ beats per minute) that includes cardizem and intravenous metoprolol injections, is prescribed. A cardiologist is consulted for possible direct-current car- 
dioversion if optimal pharmacologic therapy fails. Bridging intravenous heparin is initiated followed 3 days later with Warfarin antithrombotic therapy for patients with persistent PAF or after heart valve procedures, with target 2.5 - 3.5 of international normalized ratio.

\section{STATISTICAL ANALYSIS}

The trial is designed to enroll 400 participants, and allowing 5\% dropout rate, to provide a statistical power of $90 \%$ to detect a $50 \%$ reduction rate based on the primary outcome measure (PAF) which is estimated to be $26 \%$ in the control amiodarone alone group. Nomogram by Douglas Altman was used for calculating sample size or power of two equal sized (200 each) groups. Data are reported as percentages or mean $\pm 2 \mathrm{SEM}$ of the mean.

For comparison of baseline characteristics Student's $t$-test or Wilcoxon rank-sum test are used for continuously distributed data. The Pearson $\chi$-square test and Fisher's exact test are used to compare categorical variables. Significance is accepted at values of $P \leq 0.05$. All $P$ values are two-sided, and the $95 \%$ confidence intervals are also presented. A multivariate log regression analysis is performed to assess for any relation between minocycline and PAF after adjusting for the independent covariate risk factor predictors of PAF after cardiac surgery. Variables of interest, including the prespecified subset, significant at the $P<0.1$ level on univariate screening analysis are retained and entered for clinical relevance before including them in multivariate logistic regression statistical analysis provided they were present in at least $2 \%$ of the sample.

Comparison in a prespecified subgroup of risk factors best identified as predictors of PAF such as ejection fraction (EF) $<35 \%$, New York Heart Association (NYHA) functional class III or IV, advanced age and coexisting cardiac disease is conducted. It is hypothesized, when multivariate analysis of these condounders, that minocycline may provide unique beneficial effects in PAF control.

\section{RESULTS}

This is an ongoing trial, but at this time it is only a few subjects have been enrolled, but the current information is appearing favorable for Minocycline.

At this time 5 pairs of patients have been enrolled in the study and analyzed. All patients in the minocycline group $(100 \%)$ had no $\mathrm{AF}$, while $20 \%$ of amiodarone alone group had no AF.

\section{DISCUSSION}

Atrial fibrillation in general and PAF in particular represent two critical problems in clinical practice. In 2009, the Institute of Medicine provided a list of priority topics (listed by quartile) in comparative effectiveness research. Atrial fibrillation was considered one of the highest quartile.

It is hypothesized that the combination of minocycline and amiodarone has a superior response rate and confers greater efficacy in PAF suppression as compared with standard amiodarone therapy administered singly. This is a valuable and relevant trial,which may represent a new management solution to reduce post cardiac surgery PAF. Additionally, post operative only dosing of minocycline will improve patient eligibility. Due to the varying limitations of the previously outlined amiodarone trials using it as a sole drug prophylaxis, perioperative minocycline supplementation combined with amiodarone leading to increased PAF reduction should be considered important. The benefit to a minocycline alone arm would be very helpful, but it would have increased the cost of this trial. We plan to add this arm in future studies. Finally, this trial offers a unique mechanism of action approach that may be used in future trials evaluating other anti-inflammatory drugs to prevent PAF. Taken together, the results of this trial may be sufficient to make recommendations for a change in the method used to prevent PAF.

\section{CONCLUSION}

$\mathrm{PAF}$ is a major clinical problem in cardiac surgery centers, consequently, interest for a superior drug regimen that can reduce the occurrence of PAF even further would be greatly welcomed. The advantage to be gained by reducing the incidence of PAF after cardiac surgery makes inception of the MINAA trial an attractive goal.

\section{DISCLOSURES}

Triax pharmaceuticals provided the Minocin free of charge. The authors have nothing to disclose with regard to commercial support, and will be solely responsible for the design, conduct, analysis, drafting and editing of this study.

\section{ACKNOWLEDGEMENTS}

The authors thank Barbara Burkott, and Cristin Coulter of the Clinical Research management office, the Cardiac Intensive Care Unit nursing staff and the Anesthesia personnel who are involved in the conduct of this study.

\section{REFERENCES}

[1] Hogue Jr., C.W. and Hyder, M.L. (2000) Atrial fibrillation after cardiac operation: Risks, mechanisms, and treatment. The Annals of Thoracic Surgery, 69, 300-306. doi:10.1016/S0003-4975(99)01267-9

[2] Lahtinen, J., Biancari, F., Salmela, E., Mosorin, M., Satta, 
J., Rainio, P., Jussi Rimpiläinen, J., Lepojärvi, M. and Juvonen, T. (2004) Post operative atrial fibrillation is a major cause of stroke after on-pump coronary artery bypass graft. The Annals of Thoracic Surgery, 77, 12411244. doi:10.1016/j.athoracsur.2003.09.077

[3] Salamon, T., Michler, R.E., Knott, K.M. and Brown, D.A. (2003) Off-pump coronary artery bypass grafting does not decrease the incidence of atrial fibrillation. The $A n$ nals of Thoracic Surgery, 75, 505-507. doi:10.1016/S0003-4975(02)04305-9

[4] Mathew, J.P., Fontes, M.L., Tudor, L.C., Ramsay, R., Duke, P., Mazer, C.D., Barash, P.G., Hsu, P.H. and Mangano, D.T. (2004) A multicenter risk index for atrial fibrillation after cardiac surgery. JAMA, 291, 1720-1729. doi:10.1001/jama.291.14.1720

[5] Aranki, S.F., Shaw, D.P., Adams, D.H., Rizzo, R.J., Couper, G.S., Vander Vliet, M., Collins, J.J., Cohn, L.H. and Burstin HR. (1996) Predictors of atrial fibrillation after coronary artery surgery: Current trends and impact on hospital resources. Circulation, 94, 390-397. doi:10.1161/01.CIR.94.3.390

[6] Hogue Jr., C.W., Creswell, L.L., Gutterman, D.D. and Fleisher, L.A. (2005) American college of chest physicians guidelines for the prevention and management of postoperative atrial fibrillation after cardiac surgery. Chest, 128, 9S-16S. doi:10.1378/chest.128.2 suppl.9S

[7] Bagshaw, S.M., Galbraith, P.D., Mitchell, L.B., Sauve, R., Exner, D.V. and Ghali, W.A. (2006) Prophylactic amiodarone for prevention of atrial fibrillation after cardiac surgery; a meta-analysis. The Annals of Thoracic Surgery, 82, 1927-1937.

doi:10.1016/j.athoracsur.2006.06.032

[8] Zimetbaum, P. (2007) Amiodarone for atrial fibrillation. The New England Journal of Medicine, 356, 935-941. doi:10.1056/NEJMct065916

[9] Aasbo, J.D., Lawrence, A.T., Krishnan, K., Kim, M. and Trohman, R.G. (2005) Amiodarone prophylaxis reduces major cardiovascular morbidity and length of stay after cardiac surgery: A meta-analysis. Annals of Internal Medicine, 143, 327-336.

[10] Mooss, A.N., Wurdeman, R.L., Sugimoto, J.T., Packard, K.A., Hilleman, D.E., Lenz, T.L., Rovang, K.S., Arcidi, J.M. and Mohiuddin, S.M. (2004) Amiodarone versus sotalol for the treatment of atrial fibrillation after open heart surgery: The reduction in postoperative cardiovascular arrhythmic events (REDUCE) trial. American Heart Journal, 148, 641-648. doi:10.1016/j.ahj.2004.04.031

[11] Zebis, L.R., Chritensen, T.D., Thomsen, H.F., Mikkelsen, M.M., Folkersen, L., Sørensen, H.T. and Hjortdal, V.E. (2007) Practical regimen for amiodarone use in preventing postoperative atrial fibrillation. The Annals of Thoracic Surgery, 83, 1326-1331. doi:10.1016/j.athoracsur.2006.09.096

[12] Yagdi, T., Nalbantgil, S., Ayik, F., Apaydin, A., Islamoglu, F., Posacioglu, H., Calkavur, T., Atay, Y. and Buket, S. (2003) Amiodarone reduces the incidence of atrial fibrillation after coronary artery bypasss grafting. The Journal of Thoracic and Cardiovascular Surgery, 125, 1420-
1425. doi:10.1016/S0022-5223(02)73292-3

[13] Mitchell, L.B., Exner, D.V., Wyse, D.G., Wyse, G., Connolly, C.J., Prystai, G.D., Bayes, A.J., Kidd, W.T., Kieser, T., Burgess, J.J., Ferland, A., MacAdams, C.L. and Maitland, A. (2005) Prophylactic oral amiodarone for the prevention of arrhythmias that begin early after revascularization, valve replacement, or repair: PAPABEAR; a randomized controlled trial. JAMA, 294, 30933100. doi:10.1001/jama.294.24.3093

[14] Guarnieri, T., Nolan, S., Gottlieb, S.O., Dudek, A. and Lowry, D.R. (1999) Intravenous amiodarone for the prevention of atrial fibrillation after open heart surgery: The amiodarone reduction in coronary heart $(\mathrm{ARCH})$ trial. Journal of the American College of Cardiology, 34, 343347. doi:10.1016/S0735-1097(99)00212-0

[15] Ishida, K., kimura, F., Imamaki, M., Ishida, A., Shimura, H., Kohno, H., Sakurai, M. and Miyazaki, M. (2006) Relation of inflammatory cytokines to atrial fibrillation after off-pump coronary artery bypass grafting. European Journal Cardio-Thoracic Surgery, 29, 501-505. doi:10.1016/j.ejcts.2005.12.028

[16] Ramlawi, B., Out, H., Mieno, S., Boodhwani, M., Sodha, N.R., Clements, R.T., Bianchi, C. and Sellke, F.W. (2007) Oxidative stress and atrial fibrillation after cardiac surgery: A case-control study. The Annals of Thoracic Surgery, 84, 1166-1173. doi:10.1016/j.athoracsur.2007.04.126

[17] Ad, N., Snir, E., Vidne, B. and Golomb, E. (2001) Histologic atrial myolysis is associated with atrial fibrillation after cardiac operation. The Annals of Thoracic Surgery, 72, 688-693. doi:10.1016/S0003-4975(01)02882-X

[18] Halonen, J., Halonen, P., Jarvinen, O., Taskinen, P., Auvinen, T., Matti Tarkka, M., Hippeläinen, M., Juvonen, T., Hartikainen, J. and Hakala, T. (2007) Corticosteroids for the prevention of atrial fibrillation after cardiac surgery: A randomized controlled trial. JAMA, 297, 15621567. doi:10.1001/jama.297.14.1562

[19] Patti, G., Chello, M., Candura, D., Pasceri, V., D’Ambrosio, A., Covino, E. and Sciascio, G.D. (2006) Randomized trial of atorvastatin for reduction of postoperative atrial fibrillation in patients undergoing cardiac surgery, results of the ARMYDA-3 (Atorvastatin for reduction of myocardial dysrhythmia after cardiac surgery) study. Circulation, 114, 1455-1461. doi:10.1161/CIRCULATIONAHA.106.621763

[20] Kraus, R.L., Pasieczny, R., Lariosa-Willingham, K., Turner, M.S., Jiang, A. and Trauger, J.W. (2005) Antioxidants properties of minocycline: neuroprotection in an oxidative stress assay and direct radical-scavenging activity. Journal of Neurochemistry, 94, 819-827. doi:10.1111/j.1471-4159.2005.03219.x

[21] Scarabelli, T.M., Stephanou, A., Pasini, E., Gitti, G., Townsend, P., Lawrence, K., Chen-Scarabelli, C., Saravolatz, L., Latchman, D., Knight, R. and Gardin, J. (2004) Miocycline inhibits caspase activation and reactivation, increases the ratio of XIAP to Smac/DIABLO, and reduces the mitochondrial leakage of cytochrome-C and Smac/DIABLO. Journal of the American College of Cardiology, 43, 865-874. doi:10.1016/j.jacc.2003.09.050 
[22] Wang, J., Wei, Q., Wang, C.Y., Hill, W.D., Hess, D.C. and Dong, Z. (2004) Minocycline up-regulates Bcl-2 and protects against cell death in mithochondria. The Journal of Biological Chemistry, 279, 19948-19954. doi:10.1074/jbc.M313629200

[23] Yrjanheikki, J., Tikka, T., Goldsteins, G., Keinänen, R., Goldsteins, G., Chan, P.H. and Koistinaho, J. (1999) A tetracycline derivate, minocycline, reduces inflammation and protects against focal cerebral ischemia with a wide therapeutic window. Proceedings of the National Academy of Sciences, 96, 13496-13500. doi:10.1073/pnas.96.23.13496
[24] Alano, C.C., Kauppinen, T.M., Viader Valls, A. and Swanson, R.A. (2006) Minocycline inhibits poly (ADP-ribose) polymerase-1 at nanomolar concentrations. Proceedings of the National Academy of Sciences, 103, 9685-9690. doi: $10.1073 /$ pnas.0600554103

[25] Martinez, E.A., Bass, E.B. and Zimetbaum, P. (2005) Pharmacologic control of rhythm: American college of chest physicians guidelines for the prevention and management of postoperative atrial fibrillation after cardiac surgery. Chest, 128, 48-55.

doi:10.1378/chest.128.2 suppl.48S 